\title{
Orientation-specific aftereffects and illusions in the perception of brightness*
}

\author{
RAY OVER $\dagger$, JACK BROERSE, BORIS CRASSINI, and WILLIAM LOVEGROVE \\ University of Queensland, St. Lucia, Australia 4067
}

\begin{abstract}
Orientation-specific brightness aftereffects were found when vertical and horizontal gratings of the same space-average luminance were viewed following alternate exposure to vertical and horizontal gratings that differed in space-average luminance. The vertical test grating appeared bright following exposure to a dim vertical grating, and dim after a bright vertical grating had been viewed. This aftereffect did not occur when the adaptation gratings had been seen by one eye and the test gratings by the other eye. An orientation-specific illusion in the perception of brightness was also found, with the white sectors of a vertical grating appearing brighter against a background of horizontal lines than they did against a background of vertical lines. Both distortions imply that there are detectors in the human visual system that are conjointly tuned to luminance and contour orientation.
\end{abstract}

A number of recent studies suggest that there is conjoint processing of color and spatial information in the human visual system. In the initial demonstration, McCollough (1965) exposed Ss alternately to a vertical grating in orange light and a horizontal grating in blue light. Subsequently, in white light, the vertical grating appeared blue and the horizontal grating looked orange. $\mathrm{McC}$ ollough attributed orientation-specific color aftereffects to selective adaptation of neural detectors tuned to both wavelength and contour orientation. She argued that only detectors selective to the specific color and orientation are excited during exposure to vertical lines in orange light. Because these detectors remain adapted for a period of time after inspection, the white vertical lines presented as the test figure are signaled via vertical edge detectors sensitive to the complementary color. A similar explanation has been proposed for color aftereffects specific to the direction of image motion (Hepler, 1968; Stromeyer \& Mansfield, 1970) and to spatial frequency (Breitmeyer \& Cooper, 1972; Lovegrove \& Over, 1972; Stromeyer, 1972). In addition to spatially selective color aftereffects, it has been possible to induce color-selective spatial aftereffects. In this case, the tilt aftereffect, in which a vertical line appears tilted counterclockwise following exposure to clockwise tilted lines, is maximal when the inspection and test patterns are the same color, and is reduced when they differ in color (Held \& Shattuck, 1971; Lovegrove \& Over, 1973). Similar color selectivity is obtained with the motion aftereffects (Favreau, Emerson, \& Corballis, 1972; Lovegrove, Over, \& Broerse, 1972; Mayhew \& Anstis, 1972).

Mayhew and Anstis (1972) have suggested that there are luminance-spatial in addition to wavelength-spatial

*This study was supported by an award from the Australian Research Grants Committee. We thank Ann Maree Parker for her assistance in data collection.

$\dagger$ Requests for reprints should be sent to Ray Over, Department of Psychology, University of Queensland. St. Lucia, Australia 4067. linkages in visual processing. They reported that alternate exposure to a spiral undergoing counterclockwise rotation in dim light and clockwise rotation in light of medium intensity resulted in opposite motion aftereffects when medium stationary and dim stationary spirals were later viewed. They also noted (without providing data) that a vertical grating appeared brighter than a horizontal grating of the same space-average luminance following alternate exposure to a bright horizontal grating and a dim verticalgrating.

The aim of Experiment I was to measure orientation selectivity in the brightness aftereffect. Os were required to match vertical and horizontal test gratings in brightness following exposure to vertical and horizontal gratings that differed in space-average luminance. Measures were obtained with the adaptation and test stimuli viewed by both eyes (binocular conditions) and by different eyes (dichoptic conditions). This comparison was made because of evidence (see Over, Long, \& Lovegrove, 1973) that color-spatial conjunctions do not apply under dichoptic conditions. In particular, spatially selective color aftereffects do not demonstrate interocular transfer (McCollough, 1965; Hepler, 1968), and spatial aftereffects are not selective to color when the inspection and test patterns are viewed by different eyes (Lovegrove, Over, \& Broerse, 1972; Lovegrove \& Over, 1973).

\section{EXPERIMENT I}

\section{Method}

Subjects

The 16 undergraduate students who were tested had normal or corrected-to-normal vision.

\section{Materials and Procedure}

The adaptation and test stimuli were displayed by back projection of grating transparencies in a Gerbrands three-field tachistoscope (Model T-3B-1). During induction of the 


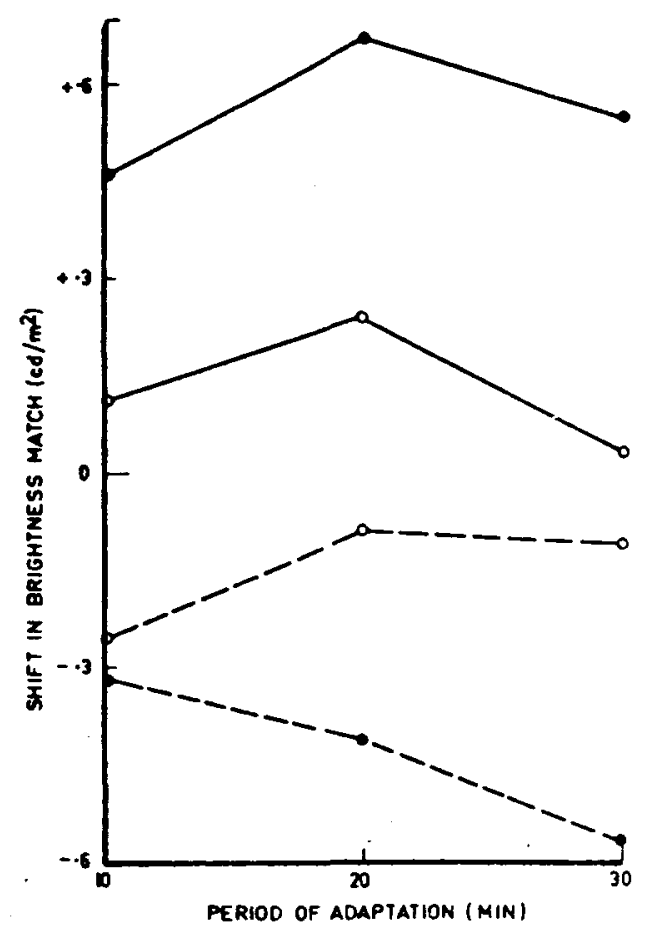

Fig. 1. Mean shift in space-average luminance needed to achieve a brightness match after 10,20 , and 30 min adaptation. Measures are given for binocular ( $\bullet$ ) and dichoptic (o) conditions, and where adaptation grating of the same orientation as the variable test grating had been $\operatorname{dim}\left(-{ }_{-}\right)$or bright (-).

aftereffect, Ss viewed vertical and horizontal square-wave gratings ( 1 cycle/deg) presented alternately every $10 \mathrm{sec}$ over a 30-min adaptation period. The space-average luminance of one grating was $9.6 \mathrm{~cd} / \mathrm{m}^{2}$, and of the other grating, $445 \mathrm{~cd} / \mathrm{m}^{2}$. Each grating subtended $4 \times 4 \mathrm{deg}$. Testing was conducted in a darkened room, and commenced after $S$ had remained in total darkness for $5 \mathrm{~min}$. The $S$ then made eight preadaptation judgments of the relative brightness of vertical and horizontal gratings, each $2 \times 4 \mathrm{deg}$, presented in separate channels of the tachistoscope and laterally juxtaposed in the field of view. The $S$ adjusted the intensity control of one channel until the white sectors of the grating in this channel matched in brightness the white sectors of the other grating (maintained at a space-average luminance of $10.9 \mathrm{~cd} / \mathrm{m}^{2}$ ). Four brightness matches were made in the same manner after 10,20 , and $30 \mathrm{~min}$ of exposure to the adaptation gratings.

Eight of the Ss viewed the adaptation and test gratings with both eyes, and the remaining eight $S s$ saw the adaptation gratings with the left eye and the test gratings with the right eye. Under each condition, the luminance and orientation combinations of the adaptation gratings were counterbalanced between $S s$, and the relative position of the test gratings as well as which test grating was taken as the standard were varied systematically within Ss.

\section{Results and Discussion}

The mean preadaptation settings made by each $S$ was taken as the baseline against which the effects of adaptation were established. Figure 1 shows the mean shift in the space-average luminance of the comparison test grating needed to maintain the brightness match with the standard test grating following different periods of adaptation. Values are shown separately for binocular and dichoptic inspection conditions, and also in terms of whether the comparison test grating was the same orientation as the dark or bright adaptation grating. An analysis of variance showed that adaptation had different effects on judgments across these four conditions, $F(3,12)=3.66, p<.05$. The mean aftereffect did not vary significantly as a function of the period of adaptation, $F(2,24)=1.89, \mathrm{p}>.05$, and the interaction between inspection conditions and the adaptation period was insignificant, $F(6,24)=0.58$, $\mathrm{p}>.05$.

Comparisons between means by Duncan's multiple range test showed that under binocular conditions the vertical comparison grating had to be made darker than the horizontal standard grating following exposure to the dark vertical grating during adaptation, and brighter following exposure to the bright vertical grating, to achieve a brightness match. Such data indicate that a vertical comparison grating appeared brighter than a horizontal standard grating of the same space-average luminance following exposure to a dark vertical grating and a bright horizontal grating. This conclusion follows because it was necessary to lower the luminance of the comparison stimulus to achieve a brightness match with the standard. Orientation-specific aftereffects in the perception of brightness were limited to binocular inspection, and did not occur when the adaptation and test gratings had been viewed by separate eyes. Measures later obtained with additional Ss showed that monoptic inspection (adaptation and test gratings viewed with the one eye) resulted in aftereffects similar to those obtained with binocular inspection. The aftereffect was reduced appreciably if, following monoptic inspection, test gratings were viewed binocularly.

Orientation-specific brightness aftereffects can be attributed to selective adaptation of neural detectors that are conjointly tuned to luminance and contour orientation. In these terms, a vertical grating would be represented in the visual system by the differential activity levels of detectors tuned to bright vertical and dark vertical lines. Exposure to a vertical grating in bright light would result in a subsequent aftereffect to the extent that one of the populations of detectors by which the test stimulus is normally signaled is in an adapted state.

\section{EXPERIMENT II}

The proposition that the human visual system contains luminance-tilt detectors leads to an expectation that the brightness of a vertical grating is affected by the orientation of contours forming the surround to the grating. It is clear from observation of Fig. 2 that the white sectors of a vertical center grating appear brighter when surrounded by horizontal than when surrounded by vertical lines, even though the center and surround 
areas are identical in space-average luminance.

Two assumptions are necessary to explain orientation selectivity in this brightness illusion: first, that brightness information is signaled by luminance-tilt detectors, and second, that inhibitory interaction occurs in visual processing only to the extent that similarly tuned detectors are stimulated by adjacent displays. Extended study of inhibitory interaction in the Limulus visual system has shown that the presence of a dimensionally relevant surround reduces input generated by a display, and there is indirect evidence (see Békésy, 1967; Ratliff, 1965) that similar inhibitory operations occur in human vision. The present claim is that the brightness of the center grating is given by the relative activity of bright vertical and dim vertical detectors, with these detectors inhibited more by vertical than by horizontal surrounding lines. The purpose of Experiment II was to measure orientation selectivity in the perceived brightness of the center grating with the tilt of the surrounds varied systematically from vertical to horizontal.

\section{Method}

Patterns of the type shown in Fig. 2 were generated by displaying, in separate fields of a Gerbrands three-field tachistoscope. a vertical grating of $3 \mathrm{deg} 8 \mathrm{~min}$ diam and an annulus with an inner diam of $3 \mathrm{deg} 8 \mathrm{~min}$ and an outer diam of $5 \mathrm{deg} 48 \mathrm{~min}$. Square-wave gratings with a periodicity of $4 \mathrm{cycles} / \mathrm{deg}$ were used. The gratings were shown simultaneously, and $S$ 's task was to adjust the intensity control of the field containing the center grating to effect a brightness match with the surround grating (maintained at $4.0 \mathrm{~cd} / \mathrm{m}^{2}$ ). The seven $\mathrm{Ss}$ had normal or corrected-to-normal vision, and each $\mathrm{S}$ made six brightness matches (three from a starting position of $5.7 \mathrm{~cd} / \mathrm{m}^{2}$ and three from $3.3 \mathrm{~cd} / \mathrm{m}^{2}$ ) at each of seven orientations (vertical to horizontal in 15-deg steps) of the surround lines. A Latin square was used to vary the order in which tilt values were tested across Ss. Viewing was monocular with the right eye. and the testing room was darkened.

\section{Results}

The mean space-average luminance necessary for the center grating to appear matched in brightness to the surround grating is shown in Fig. 3 as a function of the orientation of the surround grating. Orientation exerted significant influence over judgments, $F(6,42)=17.10$, $\mathrm{p}<.01$, and the distortion was such that when the center and surround patterns were of the same space-average luminance the white sectors of the center grating appeared brighter when the surround grating was horizontal than when it was vertical. Comparisons between means by Duncan's multiple range test showed that judgments did not change significantly $(p>.05)$ as the orientation of the surround grating was varied from vertical to $45 \mathrm{deg}$. The relative insensitivity of brightness perception to orientation relationships can be contrasted with psychophysical evidence (e.g., Fidell, 1970; Held \& Shattuck, 1971; Lovegrove \& Over, 1973). indicating that color-tilt detectors are finely tuned to orientation.

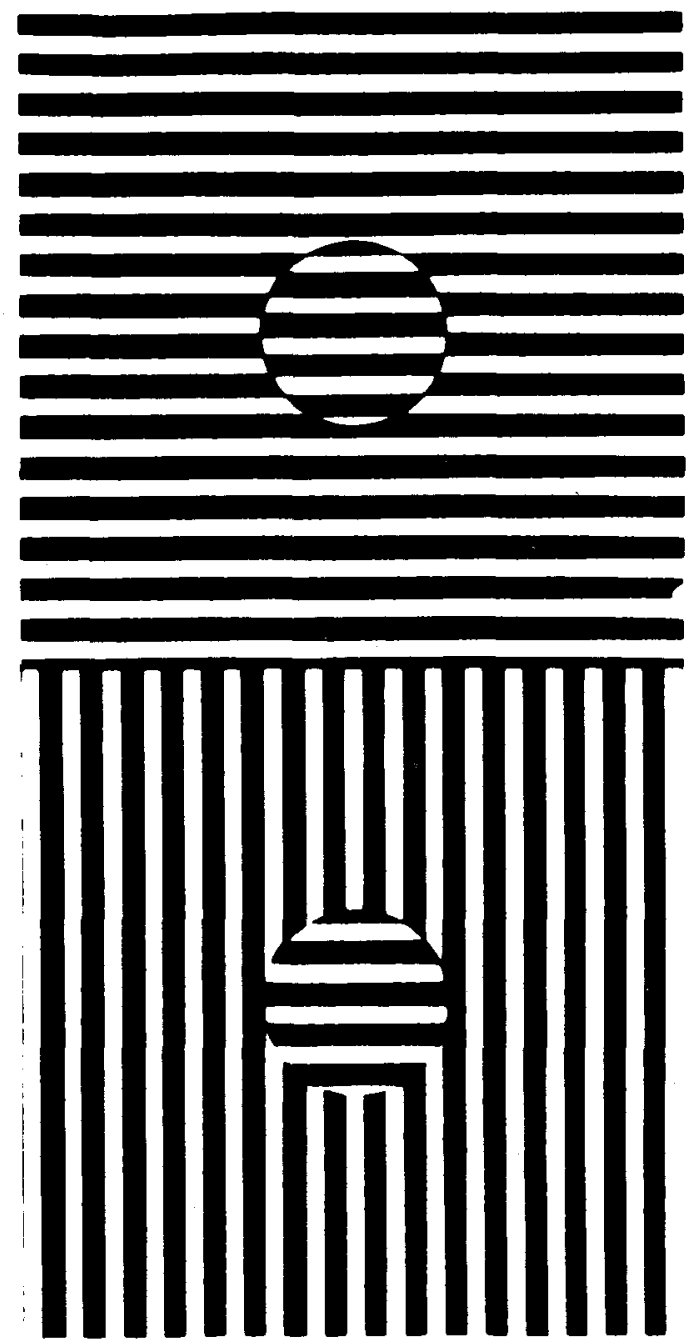

Fig. 2. The white sectors of the horizontal center grating appear brighter when surrounded by vertical than when surrounded by horizontal lines.

\section{DISCUSSION}

Mayhew and Anstis (1972) proposed that orientation-specific color aftereffects first reported by McCollough (1965) were simply one instance of a general class of perceptual aftereffect dependent on selective adaptation of double-duty detectors in human vision. Color-tilt, color-motion, and color-periodicity pairings have received systematic attention, and luminance-motion linkages have also been studied. The present experiments provide evidence of conjoint processing of luminance and contour orientation, and the data on orientation-specific brightness aftereffects can be readily incorporated within the general model proposed by Mayhew and Anstis (1972).

The demonstration of an orientation-selective brightness illusion suggests three directions of future research. The first expectation is that simultaneous brightness contrast, in which the center and surround 


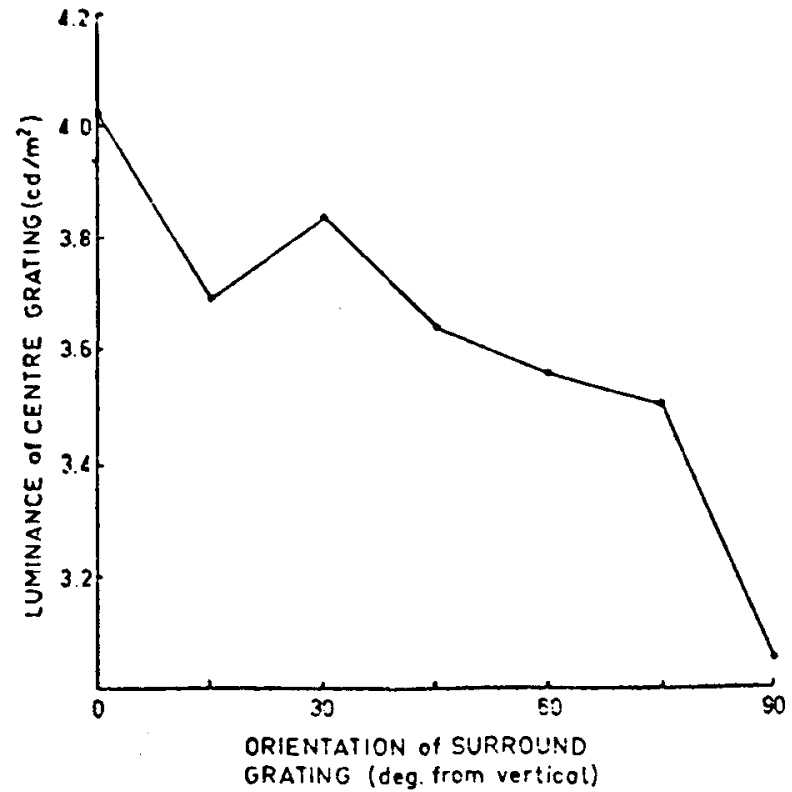

Fig. 3. Mean space-average luminance needed for center and surround gratings to appear equal in brightness.

patterns differ in space-average luminance, will be orientation-selective if gratings rather than homogeneous fields are used as the adaptation and test stimuli. Second, the color appearance of a black and white grating surrounded by a colored grating should depend on the relative orientation of the two gratings. The vertical center grating should appear more green when surrounded by red vertical than by red horizontal lines. This distortion can be explained in the same logic as the McCollough effect, except that inhibitory interaction is now occurring between simultaneously rather than successively presented stimuli. Third, orientation selectivity in color aftereffects (e.g., McCollough, 1965) is paralleled by color selectivity in orientation aftereffects (Held \& Shattuck, 1971). A question of interest is whether orientation selectivity in simultaneous color contrast is paralleled by color selectivity in simultaneous orientation contrast. The latter form of distortion is commonly referred to as geometrical illusion, and in some cases (e.g., Blakemore, Carpenter, \& Georgeson, 1970) is attributed to inhibitory interaction. Study of illusions with the inducing and test contours shown in the same and different colored light may yield clues as to the mechanism of these distortions.

Simultaneous and successive perception should be compared for one further reason. Mayhew and Anstis (1972) have attributed the extreme persistence of the McCollough effect after cessation of adaptation to the properties of the color-spatial analyzing mechanism rather than to the aftereffect process. However, evidence of orientation selectivity in simultaneous color contrast in a manner analogous to the McCollough effect would suggest that persistence in orientation-specific color aftereffects has to do with operations involved in successive display of stimuli rather than with double-duty processing. This possibility is suggested by evidence that the traditional motion aftereffect, which does not involve double-duty processing, can persist for a day or more (Masland, 1969; Kalfin \& Locke, 1972).

\section{REFERENCES}

Békésy, G. v. Sensory inhibition. Princeton: Princeton University Press, 1967.

Blakemore, C.. Carpenter, R. H. S., \& Georgeson, M. A. Lateral inhibition between orientation detectors in the human visual system. Nature, 1970. 228. 37-39.

Breitmeyer, B. G., \& Cooper, L. A. Frequency-specific color adaptation in the human visual system. Perception \&. Psychophysics, 1972, 11, 95-96.

Favreau, O., Emerson, V., \& Corballis, M. C. Movement aftereffects contingent on color. Science, 1972, 176, 78-79.

Fidell, L. S. Orientation specificity in chromatic adaptation of human "edge-detectors." Perception \& Psychophysics, 1970. $8,235-237$.

Held, R., \& Shattuck, S. R. Color and edge-sensitive channels in the human visual system: Tuning for orientation. Science, $1971,174,314-316$.

Hepler, N. Color: A motion-contingent aftereffect. Science, $1968,162,376-377$.

Kalfin, K., \& Locke, S. Evaluation of long term visual motion after-image following monocular stimulation. Vision Research. $1972,12,359-361$.

Lovegrove, W. J., \& Over, R. Color adaptation of spatial frequency detectors in human vision. Science, 1972, 176. $541-543$.

Lovegrove, W. J., \& Over, R. Color-selectivity in orientation masking and aftereffect. Vision Research, 1973, 13, 895-901.

Lovegrove, W. J., Over, R., \& Broerse, J. Color-selectivity of the motion aftereffect. Nature, 1972, 238, 334-335.

$\mathrm{McC}$ ollough, $\mathrm{C}$. Color adaptation of edge-detectors in the human visual system. Science, 1965, 149, 1115-1116.

Masland, R. H. Visual motion perception. Science, 1969, 165 , 819-820.

Mayhew, J. E. W., \& Anstis, S. M. Movement aftereffects contingent on color, intensity, and pattern. Perception \& Psychophysics, 1972, 12, 77-85.

Over, R., Long, N., \& Lovegrove, W. Absence of binocular interaction between spatial and color attributes of visual stimuli. Perception \& Psychophysics, 1973, 13, 534-540.

Ratliff, F. Mach bands: Quantitative studies on neural networks in the retina. San Francisco: Holden-Day, 1965.

Stromeyer, C. F. Edge-contingent color aftereffects: Spatial frequency selectivity. Vision Research, 1972, 12, 717-733.

Stromeyer, C. F., \& Mansfield, R. J. W. Colored aftereffects produced with moving edges. Perception \& Psychophysics, 1970, 7, 108-114.

(Received for publication February 23, 1973; revision received July $25,1973$. ) 